Abstract ID: 35

\title{
Extended reliability measures for the fast method of schroeder-phase masking
}

\author{
Sarah Rahmat ${ }^{\mathrm{a}}$ | Nur Afiqah Binti Amirullah ${ }^{\mathrm{a}}$ | Greg A. O’Beirne ${ }^{\mathrm{b}}$ \\ anternational Islamic University Malaysia \\ ${ }^{b}$ University of Canterbury, New Zealand
}

Introduction: Schroeder-phase masking test has been found to be highly useful in providing information on the cochlea phase response that was not previously discovered. Conventional Three Alternative Forced Choice (3AFC) method of conducting Schroeder phase masking takes a long testing time (around 45 minutes) and could possibly limit the valuable information that may be gathered from this test. A faster Schroeder-phase masking method which is able to cut down almost $80 \%$ of the testing time has been developed, and has been proven reliable for normal hearing population. This study aims to extend the reliability measures to hearing impaired population. Methods: This is an observational study involving 10 normal hearing and 6 sensorineural hearing loss subjects. Schroeder phase masking functions were measured at $500 \mathrm{~Hz}, 1 \mathrm{kHz}$ and $2 \mathrm{kHz}(75 \mathrm{~dB} \mathrm{~A})$ using the conventional and fast methods to find the agreement between two methods. The fast method was measured in two sessions to find the test-retest reliability. Results: The fast method showed; i) good agreement with the conventional 3 AFC method-no significant difference of masked thresholds between two methods at majority of the tested conditions $(p>0.05)$, ii) good test retest reliability- no significant difference of masked thresholds between two sessions ( $p>0.05$ ). Conclusions: Proven to be a reliable method in both normal hearing and hearing impaired population, the faster method could potentially facilitate more Schroeder phase masking experiments in investigating the fundamental of sound processing and auditory perception.

KEYWORDS: Cochlear phase response; Psychoacoustic; Fast method; Schroeder phase masking 